\title{
Multi-objective optimization method for the ATO system using Cellular Automata
}

\author{
J. Xun, B. Ning \& K. P. Li \\ The Key State Laboratory of Rail Traffic Control and Safety, \\ Beijing Jiaotong University, Beijing, People's Republic of China
}

\begin{abstract}
Automatic Train Operation (ATO) is one of the most important functions for an advanced train control system in high-speed railway systems. Research on optimization methods for ATO has been done before it is implemented in a train control system. From a theoretical point of view, it can be formulated as one of the functions of multi-objective Optimal Control Theory. This paper presents a new multi-objective optimization method for an ATO system using Cellular Automata (CA). A CA model for an ATO system is applied to simulate train operation. An optimal method for ATO is proposed. Compared with actual train operation results, the control algorithm can reduce energy consumption and ensure train operation safety such as higher accuracy of train stop. Therefore, it can improve the efficiency and safety of the train operation.
\end{abstract}

Keywords: Automatic Train Operation (ATO), Cellular Automata (CA), optimal method.

\section{Introduction}

ATO system has been known as one of the most effective methods for saving energy and improving the transportation efficiency. Along with the rapid improvement of technologies, especially with the development of CBTC (Communication Based Train Control) system [1], more and more ATO systems have been put in operation. The control algorithm is the core of ATO system. Many scholars did a lot of researches on ATO control algorithm. C.S.Chang proposed a novel approach of differential evolution (DE) by incorporating the Pareto-optimal set which is presented for optimizing train movement through tuning fuzzy membership functions in mass transit systems [2]. Satoshi Sekine 
proposed two-degree-of-freedom fuzzy neural network control systems [3]. Tang Tao presented the basic structure and functions of ATO system [4].

At present, there are several systems with ATO function from the different companies in the world. For instance, TRAINGUARD from Siemens has been used in M4 line and M5 line in Guangzhou Metro in China since 2004. The SELTRAN from Alcatel has been used in light rail in Wuhan Metro and M3 line in Guangzhou Metro. URBALIS from Alstom has been used in M2 line and Airport line for Beijing Metro. There are also ATO systems from Hitachi and Westinghouse etc.

Some scholars have put Cellular Automata into analyzing train movement. Li Ke-Ping et al. [5] and Ning Bin et al. [6] extended the NaSch model and proposed an improved model of train-following in rail system. Zhou Hua Liang et al. [7] simulated the traffic phenomenon of delay propagation using a cellular automaton traffic model for moving-like block system. In [8], a CA model that extended to the railway network was proposed.

What we present in this paper is about the researches on a new multiobjective optimization method for ATO system using cellular automata. We simulate some train trajectories with different optimal objects and discuss the influence among different optimal objects.

\section{Automatic Train Operation system}

Automatic Train Control system includes ATP (Automatic Train Protection), ATS (Automatic Train Supervision) and ATO (Automatic Train Operation). ATP is the safety system which ensures that trains remain a safe distance and have sufficient warning to allow them to stop without colliding with another train. The ATS can help dispatcher supervise and manage train operation. ATO (Automatic Train Operation) is concerned on the parts of train operation related to station stops and starts, punctuality, comfort and energy saving. It will receive commands from ATS to adjust train speed. The running speed can not exceed the speed restriction by ATP. Without ATP and ATS, ATO can't work independently.

The schematic diagram is shown as Figure 1.

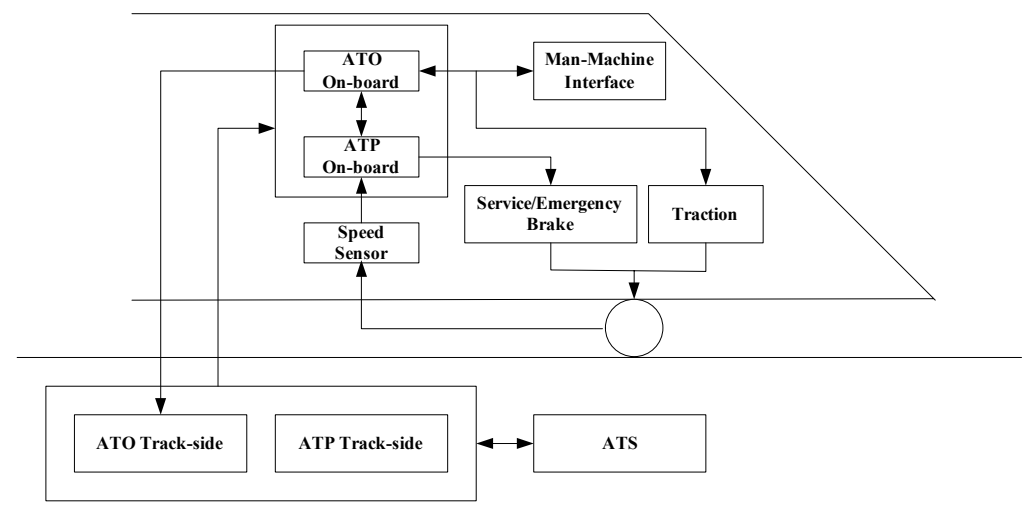

Figure 1: The schematic diagram of ATO system. 
ATO controller should consider the train parameters and line parameters, such as slope and curve etc. According to schedule and real-time information, it decides the brake or propulsion rate of train. Under the limitation of ATP, ATO controller will gather the information which is helpful to its decision, such as train speed, programmed stop and dwell time, and so on. Figure 2 shows the ATO controller model.

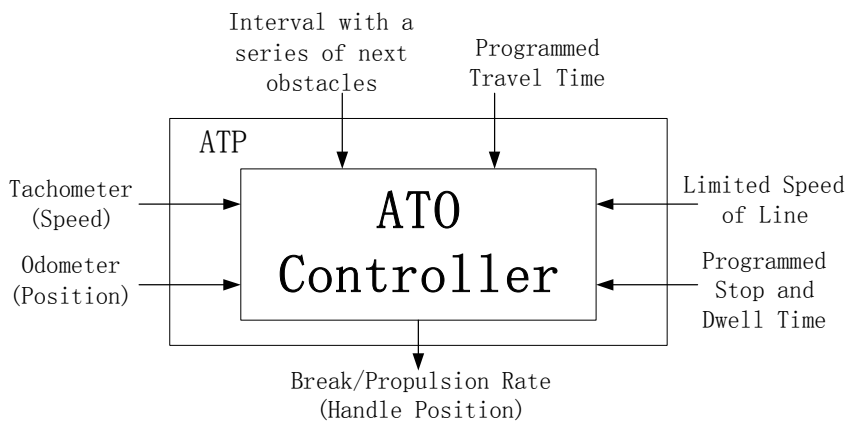

Figure 2: $\quad$ ATO controller model.

In the development of ATO, the control algorithms which have been used can be put into 4 types [4]:

(1) First type: PID (Proportion-Integral-Derivative) Control. Because of its simple and convenient operation, the ATO system with PID control algorithm is firstly put into subway train control. This method belongs to classical control method. The core is to compute based on the traction/braking character formulae, and then to control train operation under different modes of start-up, coasting, traction and braking modes. Because of the low demand of traffic at that time, the ATO system which is controlled by this method is put in operation in several lines.

(2) Second type: Parameter Self-adaptive Control. Since some of uncertain factors affect ATO system, the control strategy will be different as the change of work condition. The traditional PID control is difficult to be used to control the complicated operation. PID control algorithm is improved, and Parameter Selfadaptive Control Algorithm appears. This method adopts adjustment strategy with adaptive parameters to decrease the influence of environment changing. The core of this method is the same with one of classical PID control.

(3) Third type: Intelligent Control. As the development of control theories, intelligent technology is applied to solve control problems. The combination of experience and intelligent control theory brings a new algorithm: intelligent control algorithm. Intelligent control system includes fuzzy control, expert system, neural network control, and so on. Fuzzy control can effectively control a system which is difficult to build a mathematical model and can be controlled with experience. Expert system can make decision with commonsense of experienced driver and unique intelligent behaviors. Neural network has the ability to parallel processing and self-learning. 
(4) Fourth type: Integrated Intelligent Control. Along with increasing requirement on ATO, single intelligent control method can't meet the requirement of ATO system. Integrated intelligent control is under consideration. Because each intelligent control algorithm has its own characteristics, several intelligent control algorithms can complement each other and achieve better results. Integration of several intelligent control algorithms is the research direction of ATO control algorithm.

ATO is a complex train control system. ATO control algorithm should consider environmental factors to decide control parameters.

\section{Optimal method with CA model}

\subsection{CA model}

Nagel and Schreckenberg developed a one-dimensional probabilistic CA model (called NaSch model), which is a model of traffic flow on a single-lane [5]. In NaSch model, the road is divided into $L$ cells numbered by $i=1,2, \quad L$, and time is discrete. Each site can be either empty or occupied by a vehicle with integer speed $V_{i}=0,1, \quad V_{\max }$, where $V_{\max }$ is the maximum speed. $X_{i}(t)$ is the position where vehicle $i$ is at time $t$, and $\operatorname{gap}_{i}(t)=X_{i+1}(t)-X_{i}(t)-1$ expresses the gap between vehicle $i$ and $i+1$ at time $t$. The underlying dynamics of NaSch model is governed by the updated rules applied at discrete time steps. All sites are simultaneously updated according to four successive steps:

(1) Acceleration: $V_{i}(t+1) \longrightarrow \min \left(V_{i}(t)+1, V_{\max }\right)$

(2) Slowing down: $V_{i}(t+1) \longrightarrow \min \left(V_{i}(t), \operatorname{gap}_{i}(t)\right) \quad\left(\operatorname{gap}_{i}(\mathrm{t})\right.$ is the number of empty cells in front of the vehicle $i$ ).

(3) Randomization: $V_{i}(t+1) \longrightarrow \max \left(V_{i}(t)-1,0\right)$ (decrease $V_{i}$ by 1 with randomization probability $p$ if $\left.V_{i}>0\right)$.

(4) Movement: $X_{i}(t+1) \longrightarrow X_{i}(t)+V_{i}(t+1)$

In the proposed model, the time interval between two sequential cellular states is 1 second. And in order to determine stop accuracy, the length of each cell corresponds to $1 \mathrm{~cm}$.

\subsection{Optimal control algorithm}

The basic requirement of ATO is to adjust train speed in operation and make train stop accurately at a station when the train approaches the station. ATO should not only adjust train speed to reach the requirement of schedule, but also achieve excellent performance in punctuality, comfort and energy saving and so on.

The following points are the main ones for the ATO performance.

\section{High-Efficiency}

This is the oriental purpose for using ATO system. ATO can improve the efficiency of the whole system and increase the capacity of line. 


\section{Punctuality}

This point is an important character for railway traffic, especially for urban mass transport. Trains run under the schedule. When a train is delayed, it needs ATO to adjust train's speed and prevent train from disordered operation.

\section{Energy-Saving}

The energy cost of the manual operation is usually more than automatic operation. Therefore, energy-efficient train control for ATO is an important approach to saving energy. The minimum of energy cost is the optimality criterion.

We obtain the optimality criterion:

$$
\min J=\min F \bullet S=\min M \int_{0}^{D} a(t) d s
$$

Because of the discrete model, eqn. (1) can be presented in a discrete form:

$$
\begin{aligned}
\min J & =\min M \sum_{0}^{D} \frac{V(t)-V(t-1)}{\Delta t} \Delta s \\
& =\min M \sum_{1}^{T}(V(t)-V(t-1)) V(t)
\end{aligned}
$$

Where $J$ is energy consumption which does not include auxiliary power needed by train, $F$ is traction force, $M$ is train weight, $a(t)$ is the acceleration at time $t, V(t)$ is the train velocity at time $t, D$ is the total length of line and $T$ is the train travel time.

\section{Comfort}

In order to improve comfort, the change of acceleration and deceleration should be infrequent and the value should be small. The total number and the maximum of acceleration and deceleration during a trip is the optimality criterion.

\section{Stop-Accuracy}

For urban mass transport, stop-accuracy is also an important character, especially for stations with PSD (Platform Screen Door). Inaccurate stop will obstruct passengers taking on and off.

It is important to tune the ATO according to the above points and also difficult since the trade-off among the above points. So the ATO system is a multiobjective control system. We can build up the objective set including Efficiency(OB_Eff), Punctuality(OB_Punc), Comfort(OB_Comf), Stop Accurancy(OB_SA) and Energy Saving(OB_ES) as follows:

$$
\text { ObjectiveS et } \quad\left\{\begin{array}{l}
\text { Efficiency (OB_Eff), } \\
\text { Punctualit y(OB_Punc) } \\
\text { Comfort(OB_Comf) } \\
\text { Stop Accurancy(OB_SA) } \\
\text { Energy Saving(OB_ES) }
\end{array}\right.
$$




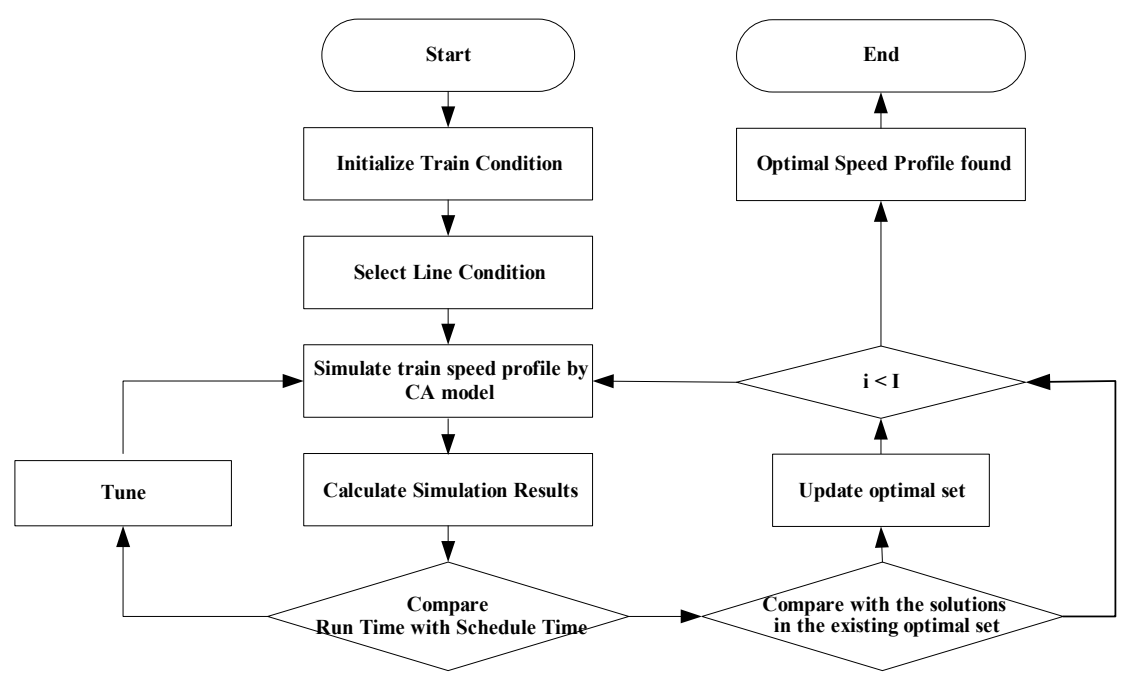

Figure 3: The flow chart of optimal algorithm.

It may be necessary to emphasize time schedule when in flat-out run optimization, or to emphasize efficiency in optimal train-coast run, and so on. Before train starts, it should be decided which is the most important object. The detailed steps of the proposed method with CA model are listed below:

Step 1. Firstly, initialize train and line condition and set $i=1$, where $i$ is the index of iteration.

Step 2. Then generate a solution according to the weights of each object.

Step 3. Simulate train speed profile with CA model and calculate the travel time, stop accuracy, the value of energy consumption, total number and maximum of acceleration and deceleration during this trip.

Step 4. Check whether the travel time of this solution is smaller than the schedule time. If yes compare with the solutions in the existing optimal set. If the new solution dominates any one in the set, then replace the old one and update the set. If no, keep the optimal set unchanged.

Step 5. Tune this solution to decrease the travel time and simulate again.

Step 6. Repeat Step 2 to 5 until $i>I$ where $I$ is the presetting number of iteration.

Step 7. If $i>I$, terminate calculation and output the results.

\section{Simulation results and its analysis}

In order to prove the validity of proposed CA model, a track in a test line between two stations NS (the North Station) and SS (the South Station) is chosen for an interstation optimization. The total length is $870.29 \mathrm{~m}$ and the maximum safety speed is $50 \mathrm{~km} / \mathrm{h}$. There are two civil speed limitations of $35 \mathrm{~km} / \mathrm{h}$ from $0 \mathrm{~m}$ to $60 \mathrm{~m}$ and from $810.29 \mathrm{~m}$ to $870.29 \mathrm{~m}$. The effect caused by the slope and curve of railway section is ignored. 
Parameters in this method are chosen as follows:

1) The maximum acceleration and deceleration a $\max =1 \mathrm{~m} / \mathrm{s}^{2}$;

2) The length of the train is $60 \mathrm{~m}$ (3 Carriages);

3) The weight of the train is $90 \mathrm{t}$ (3 Carriages);

4) The deceleration caused by air resistance and friction between track and wheel is considered as $0.04 \mathrm{~m} / \mathrm{s}^{2}$;

5) The scheduled time runtime is $100 \mathrm{~s}$.

Three cases with different optimal subjects are studied in the simulation. The data of first three cases in Table 1 are obtained from simulation and the data of the fourth case are actual train running results (Energy Consumption is computed by equation (2) and not actual train running energy cost). The results in Table 1 show that the train performance has been much improved after optimizing with the proposed method. In case 2, the proposed method not only improves the comfort of passengers but also ensures that the train finishes its trip on time. Assume all the regenerated energy is fully absorbed by other train, the train in case 2 performs the optimal object in Energy-Saving and Comfort. Otherwise, the train in case 3 reaches the destination with the shortest travel time. In case 2 and 3 , the distance between actual stop and the stop sign is only $0.02 \mathrm{~m}$. It's a great increase compared with actual experimental stop accuracy in case 4 . The effect of communication between train and trackside will be improved and the train safety will be strengthened.

Table 1: $\quad$ Comparison of train performances.

\begin{tabular}{|c|c|c|c|c|c|}
\hline \multirow{2}{*}{$\begin{array}{l}\text { Case } \\
\text { ID }\end{array}$} & \multicolumn{2}{|c|}{ Acceleration } & \multirow{2}{*}{$\begin{array}{l}\text { Travel } \\
\text { Time(s) }\end{array}$} & \multirow{2}{*}{$\begin{array}{c}\text { Energy } \\
\text { Consumption(MJ) }\end{array}$} & \multirow{2}{*}{$\begin{array}{c}\text { Stop } \\
\text { Accuracy }(m)\end{array}$} \\
\hline & $\begin{array}{c}\text { Total } \\
\text { Count }\end{array}$ & $\begin{array}{c}\text { Maximum } \\
\left(\mathrm{m} / \mathrm{s}^{2}\right)\end{array}$ & & & \\
\hline 1 & 8 & 1.24 & 92 & 195.03 & -0.02 \\
\hline 2 & 4 & 1.00 & 99 & 164.87 & -0.02 \\
\hline 3 & 8 & 1.26 & 91 & 201.52 & -0.03 \\
\hline 4 & 8 & 1.38 & 94.215 & 402.96 & 0.14 \\
\hline
\end{tabular}

Case 1: typical condition (Fig. 4a-4b)

Compared with the case 2 and 3, the present case is well balanced in terms of all the objectives. For example, it use less energy, improve the passenger comfort and runs only slightly slower than the flat-out run condition $(92$ seconds to complete the trip). Compared with the case 2 , it greatly decreases the travel time with the energy consumption increasing. This is a typical case taken from the final optimal set.

\section{Case 2: least energy condition (Fig. 4c-4d)}

When the train accelerates close to the civil speed limit, it will cut off the traction and start to coast. Then train will coast for a few seconds until it has to take breaking to stop at the station SS. The energy consumption during this trip is the least in all cases. 


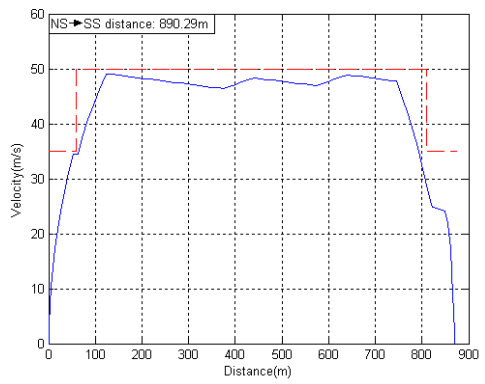

(a)

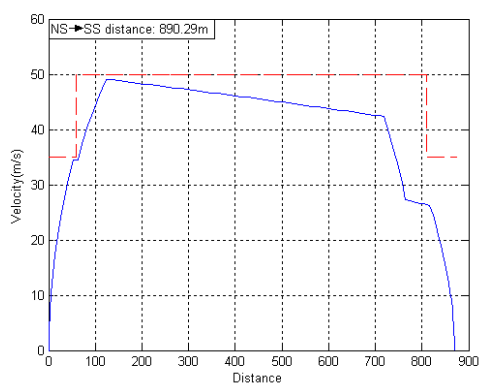

(c)

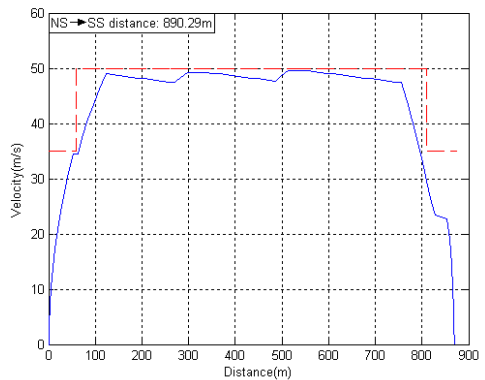

(e)

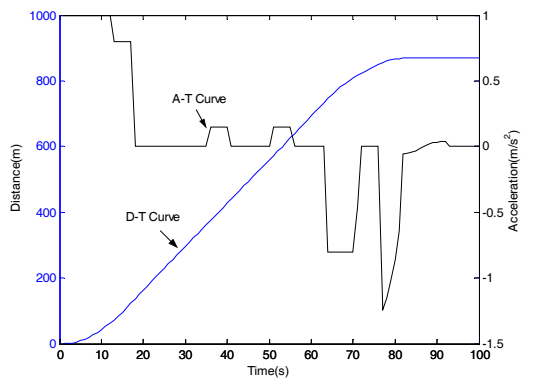

(b)

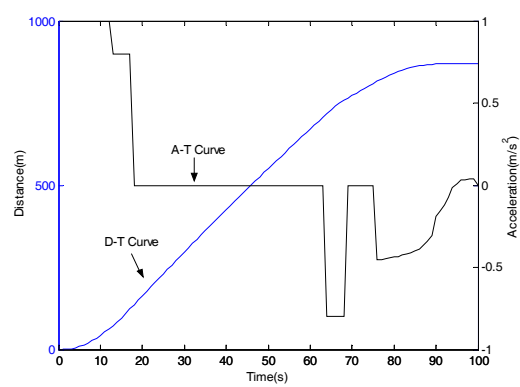

(d)

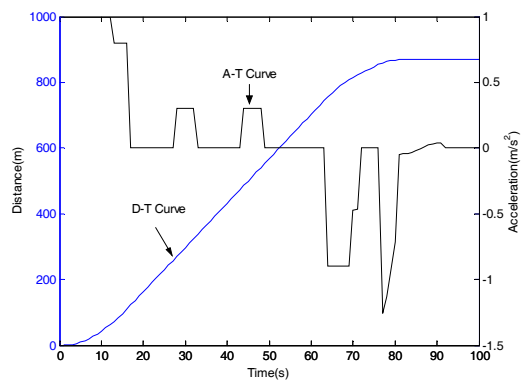

(f)

Figure 4: The result of simulation with different object.

\section{Case 3: flat-out condition (Fig. 4e-4f)}

The train is seen to run at the highest possible speed with the velocity profile very close to the civil speed limit. It takes only 91 seconds to finish the entire travel with average speed reaching a maximum value of $34.43 \mathrm{~km} / \mathrm{h}$.

The comparison between the simulation results and the actual train operation results is shown in Figure 5. The solid line denotes the simulation values using the proposed method, and the dotted line denotes the actual train values gained from tachometer. From Figure 5, we can see that the simulation values are close to the actual values. At acceleration region, the simulation results and actual 
results are nearly equal. There is a margin at deceleration region and the simulation values exceed the actual values. These results demonstrate the dependability and availability of the method.

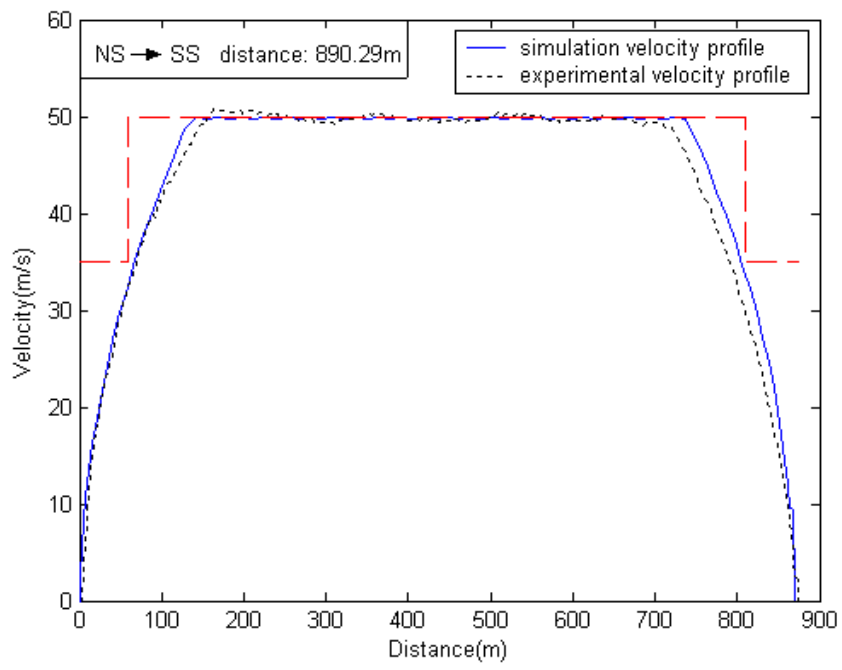

Figure 5: The comparison between simulation data and actual experimental data.

\section{Conclusions}

An ATO system can not only adjust train speed in operation but also achieve excellent performance in punctuality, comfort and energy saving and so on. This paper proposes a new multi-objective optimization method for ATO system using cellular automata. The simulation results demonstrate that the train performances are really improved after optimizing with the proposed method. The comparison between actual train operation results and simulation results is also discussed. Finally the dependability and availability of the method is proved initially.

The proposed method is preliminary and the simulation results are proved to be effective. However since it does not consider slope and curve of the line, the future research should focus on improving the proposed method by considering the effect caused by more parameters such as slope, curve and so on. The method will be further perfected.

\section{Acknowledgements}

The project is supported by the National Natural Science Foundation of China under Grant No. 60634010, the Key Project of Chinese Ministry of Education under Grant No. 107007 and Beijing Area Key Laboratory Urban Rail Transport Automation and control. 


\section{References}

[1] Ning Bin, Tang Tao, Qiu Kuan Min and Gao Chun Hai "CBTC(Communication Based Train Control): system and development" Computers in Railways X WIT Press pp 413-420 July 2006, Comprail 2006, Prague, Crech Republic.

[2] C.S.Chang, D.Y.Xu and H.B.Quek "Pareto-optimal set based multiobjective tuning of fuzzy automatic train operation for mass transit system" IEE ProcElectr. Power Appl., Volume 146, No. 5, September 1999, pp. 577-583.

[3] Sekine, S., Imasaki, N. and Endo, T. "Application of fuzzy neural network control to automatic train operation and tuning of its control rules" Fuzzy Systems, 1995. International Joint Conference of the Fourth IEEE International Conference on Fuzzy Systems and the Second International Fuzzy Engineering Symposium., Proceedings of 1995 IEEE International Conference on, Volume 4, 1995, pp. 1741-1746.

[4] Tang Tao and Huang Liang-ji "A Survey of Control Algorithm of Automatic Train Operation" Journal of the China railway Society, Volume 25, No.2, 2003 pp. 98-102.

[5] Li Ke-Ping, Gao Zi-You and Ning Bin "Cellular automaton model for railway traffic" Journal of Compute Physics, Volume 209, 2005, pp 179192.

[6] Ning Bin, Li Ke-Ping and Gao Zi-You "Modeling fixed-block railway signaling system using cellular automata model" International Journal of Modern Physics, Volume16, 2005, pp. 17931801.

[7] Zhou Hua-Liang, Gao Zi-You and Li Ke-Ping "Cellular automaton model for moving-like block system and study of train's delay propagation" Acta Phys. Sin., Volume 55, 2006, pp. 1706-1710.

[8] Xun Jing, Ning Bin and Li Ke-Ping "Network-based train-following model and study of train's delay propagation" Acta Phys. Sin., Volume 56, September 2007, pp. 5158-5164. 